\title{
On the spectroastrometric separation of binary point-source fluxes
}

\author{
John M. Porter ${ }^{1}$, R. D. Oudmaijer ${ }^{2}$, and D. Baines ${ }^{2,3}$ \\ 1 Astrophysics Research Institute, Liverpool John Moores University, Twelve Quays House, Egerton Wharf, \\ Birkenhead CH41 1LD, UK \\ e-mail: jmp@astro.livjm.ac.uk \\ 2 School of Physics and Astronomy, University of Leeds, Leeds LS2 9JT, UK \\ 3 Astrophysics, University of Oxford, Denys Wilkinson Laboratory, Keble Road, Oxford OX1 3RH, UK
}

Received 14 November 2003 / Accepted 11 August 2004

\begin{abstract}
Spectroastrometry is a technique which has the potential to resolve flux distributions on scales of milliarcseconds. In this study, we examine the application of spectroastrometry to binary point sources which are spatially unresolved due to the observational point spread function convolution. The technique uses measurements with sub-pixel accuracy of the position centroid of high signal-to-noise long-slit spectrum observations. With the objects in the binary contributing fractionally more or less at different wavelengths (particularly across spectral lines), the variation of the position centroid with wavelength provides some information on the spatial distribution of the flux. We examine the width of the flux distribution in the spatial direction, and present its relation to the ratio of the fluxes of the two components of the binary. Measurement of three observables (total flux, position centroid and flux distribution width) at each wavelength allows a unique separation of the total flux into its component parts even though the angular separation of the binary is smaller than the observations' point-spread function. This is because we have three relevant observables for three unknowns (the two fluxes, and the angular separation of the binary), which therefore generates a closed problem. This is a wholly different technique than conventional deconvolution methods, which produce information on angular sizes of the sampling scale. Spectroastrometry can produce information on smaller scales than conventional deconvolution, and is successful in separating fluxes in a binary object with a separation of less than one pixel. We present an analysis of the errors involved in making binary object spectroastrometric measurements and the separation method, and highlight necessary observing methodology.
\end{abstract}

Key words. techniques: high angular resolution - techniques: spectroscopic - stars: binaries: general

\section{Introduction}

A telescope is limited by its resolution: traditionally, the smallest resolvable feature will typically be the size of the point-spread function (PSF). There are several contributions to the PSF including the Earth's atmosphere (via seeing) for ground based telescopes, and the telescope itself (via its optics). Conventionally, if the physical size of the flux distribution is smaller than the PSF, then all the information on that scale is blended and hence cannot be retrieved. For the special case of a binary object, there have been several techniques which have been proposed to extract the individual binary star spectra from the composite spectrum, involving single value decomposition (Simon \& Sturm 1994), Fourier methods (e.g. Hadrava 1995), or Doppler tomography (Bagnuolo et al. 1992).

The technique of spectroastrometry allows the observer to gain some information on the distribution of flux on a spatial scale smaller than the PSF. It was originally discussed in the 1980s by Beckers (1982), and Christy et al. (1983) with more recent studies by Bailey (1998a,b), Garcia et al. (1999),
Takami et al. (2001, 2003). Given that the technique has the potential to provide information about the flux distribution on milliarcsecond scales, it is surprising that it has not been more widely exploited.

Conceptually, spectroastrometry is easy to grasp: it involves taking a long slit spectrum, and relies on the observer being able to determine the centroid of a flux distribution to a fraction of a pixel. The exact spatial centre of a flux distribution may vary with wavelength if the components making up that distribution contribute differing fractions of the total flux at a given wavelength. For example, a pair of objects in a binary system contribute different amounts of flux at different wavelengths. Where one object has an emission line, the position centroid of the flux will move toward that object across that line before returning to the continuum position. For an absorption line, the opposite occurs. If the centroid position can be located accurately enough, then the relative contribution of the two objects may be calculated. Any astronomical source with a flux distribution which is asymmetric with respect to wavelength may be subject to the technique, such as binary stars 
(e.g. Bailey 1998a,b), or outflows and discs (e.g. Takami et al. 2003).

More familiar techniques of deconvolution of long slit spectra have been presented by Courbin et al. (2000). Courbin et al. produce final results which are sampled at the Nyquist frequency of the observation (determined by the CCD chip pixels, see the discussion in Sect. 2 of Magain et al. 1998). Therefore, these techniques provide a method of producing a higher resolution than the observations, although they cannot provide any information on scales smaller than the Nyquist scale.

Lucy \& Walsh (2003, and references therein) produce an iterative technique, which is applied to the extraction of stellar spectra from a (crowded) convolved image. This is able to separate fluxes from objects with overlapping PSF by nominating a priori the objects which are point sources. This procedure performs well in crowded fields, but is not sensitive to objects so close that their separation is smaller than the PSF.

Here, we concentrate on a method of the separation of a "discrete" flux distribution - a binary object - into its components. Our aim is to produce a method which is able to extract the two fluxes from a binary object which is separated by less than a pixel, (an achievement impossible with previously published methods) whilst remaining competitive for larger separations when compared to more traditional deconvolution techniques.

The current observational technique of spectroastrometry (photocentre displacement) is supplemented by an investigation into the width of the flux distribution and this provides the raison d'être of this study: the addition of the information provided by the flux distribution's width closes the set of observables (total flux, photocentre position, and width of flux distribution) and unknowns (the two spectra and their separation) and thereby enables an unique separation of the composite spectrum into its component parts. We leave the problem of continuous flux distributions (e.g. a disc) until a later study (spectroastrometry of a continuous flux distribution will yield the sub-pixel scale kinematics of the source).

In Sect. 2 the binary object flux distribution its statistical properties are examined, before illustrating the flux separation technique in Sect. 3. To provide a practical guide for this technique, a discussion of errors is provided in Sect. 4 and optimal observing strategies are given in Sect. 5. Discussion and conclusions are given in Sects. 6 and 7 respectively.

\section{Observational statistics of the flux distribution of a convolved binary object}

The positional distribution of flux from any source is labelled as $\mathcal{F}_{\lambda}(x)$, where $\lambda$ is wavelength and $x$ is the position along the slit. If the source is now assumed to be a binary object with a separation of many times the radius of either object, then the positional distribution of the flux is approximately

$\mathcal{F}_{\lambda}(x)=f_{1, \lambda} \delta_{x, x_{1}}+f_{2, \lambda} \delta_{x, x_{2}}$,

where $f_{1, \lambda}$ and $f_{2, \lambda}$ are the fluxes of the two objects located at positions $x_{1}$ and $x_{2}$ respectively and $\delta_{a, b}$ is the Kronecka delta function.
We imagine that the flux is observed with a spectrometer and detected with a CCD detector with dimensions $n \times m$ pixels. The flux is sampled at discrete positions and wavelength: the pixel number in the spatial direction is labelled with index $i$, with $i=0$ corresponding to the bottom of the CCD frame and $i=n$ corresponding to the top of the frame. Likewise the pixels in the dispersion (wavelength) direction are labelled with index $j$ with $0 \leq j \leq m$. The flux in each CCD pixel is labelled $F_{i, j}$.

During an observation, the flux distribution $\mathcal{F}_{\lambda}(x)$ is first convolved with a function taking into account the seeing profile (for ground based telescopes), and telescope optics. We label this intrinsic "seeing" function $S_{\lambda}(x)$. The flux is then binned into pixels as it is detected by the CCD. The measured distribution $F_{i, j}$ is then

$F_{i, j}=\operatorname{discretize}\left[\mathcal{F}_{\lambda}(x) \otimes S_{\lambda}(x)\right]$

We label the intrinsic width of the seeing function as $\sigma_{\mathrm{S}}$, which in the case of a Gaussian function is equivalent to the standard deviation. If the separation between the objects $\left|x_{1}-x_{2}\right|$ is less than $\sigma_{\mathrm{S}}$, then the two objects are formally not resolved. Conventionally, separation of the individual spectra cannot be achieved.

Observations have two main sources of noise (i) photon counting errors, characterised by Poisson statistics of the number of photons in each pixel, and (ii) read noise generated in the process of charge transfer in the CCD during the read out stage. The relative contribution of these two error sources is dependent on the exposure time and brightness of the source observed, as well as the spectrometer itself. First we consider the case of zero noise to develop the fundamental principles and technique, and we return to the effects of noise later.

The three simple observables which may be measured from the flux distribution on the CCD are the total flux, the mean position of the flux, and the width of the flux distribution. In order to make the discussion as general as possible, henceforth we work with units of pixels.

\subsection{Total flux}

At a given wavelength $\lambda$, corresponding to pixels $(i=1 \cdots n, j)$ the flux is distributed in position according to Eq. (1) and sampled onto pixel row $i$ of the CCD. The "extracted" total flux is the sum over all pixels in the spatial direction of the flux in each pixel: $\sum_{i=0}^{n} F_{i, j} \equiv f_{\text {tot }, j}=f_{1, j}+f_{2, j}$. In traditional spectrographic work, this total extracted flux $f_{\text {tot }, j}$ (the spectrum) is the sole quantity gleaned from the observation.

\subsection{Position centroid}

The position of a distribution is often measured by the mean of the distribution - the 1 st moment. However, it is often the case that the mean is not particularly robust for non-Gaussian distributions (especially when noise is present, see e.g. Beers et al. 1990), and so a more sophisticated approach is warranted. Good choices are the M-estimators such as Tukey's biweight, or Andrew's Sine (or wave) estimators (Goodall 1983; 
Press et al. 1986). These have a weighting function which penalises outliers from the distribution, which in this context translates to estimating the width over a specific pixel region. This will then avoid problems with extended wings of seeing profiles (and also cosmic ray hits, and noise-generated errors).

We denote the position centroid of the distribution (however it is measured) as $\mu_{j}$ : note that it can be measured to fractions of a pixel hence $\mu_{j}$ is a real number and not an integer. How does the centroid $\mu_{j}$ vary as the ratio of the flux of the two objects varies? This is the aspect of spectroastrometry which has received the most attention (Bailey 1998a,b; Garcia et al. 1999; Takami et al. 2001, 2003). For equal fluxes $f_{1, j}=f_{2, j}$ the centroid will lie exactly in the middle of flux distribution (at $\left.\left(x_{2}-x_{1}\right) / 2\right)$.

The position centroid $\mu_{j}$ is a direct probe of the relative contribution of the two fluxes to the total flux. A simple relation can be written to calculate $\mu_{j}$ in terms of the two fluxes:

$\mu_{j} \equiv \frac{f_{1, j} \delta_{x, x_{1}}}{f_{\mathrm{tot}, j}}+\frac{f_{2, j} \delta_{x, x_{2}}}{f_{\mathrm{tot}, j}}$,

(e.g. Bailey 1998a) assuming that both point sources are in the spectrometer slit.

A particularly useful spectral feature which is used in the deconvolution technique below is an emission/absorption line. These push the centroid toward the brighter object over a small wavelength range - clear in the centroid spectra of Mira (Fig. 2 of Bailey 1998a) and HK Ori (Fig. 9 of Baines et al. 2004). Any slowly varying changes in the centroid can be subtracted out producing a centroid spectrum $\mu_{j}^{\prime}$ which is calibrated to be zero away from the line centre (in the continuum) and may be written as:

$\frac{\mu_{j}^{\prime}}{d} \equiv \frac{f_{2, j}}{f_{\text {tot }, j}}-\frac{f_{2, \mathrm{cont}}}{f_{\text {tot,cont }}}=\frac{r_{j}}{r_{j}+1}-\frac{r_{\text {cont }}}{r_{\text {cont }}+1}$,

(see Eq. (3) of Takami et al. 2003), where $f_{2, \text { cont }}$ and $f_{\text {tot,cont }}$ are the continuum fluxes of object 2 and the total flux, and $d$ is the separation of the objects in pixels. The final equality in Eq. (4) is expressed in terms of the ratio of the fluxes $r_{j}=f_{2, j} / f_{1, j}$ and the flux ratio in the continuum $r_{\text {cont }}=f_{2 \text {, cont }} / f_{1 \text {, cont }}$ which is used later in Sect. 3.

\subsection{Width of the flux distribution}

The width of a distribution may be measured using several methods: the simplest characteristic width of the flux distribution is the mean absolute deviation, and the most commonly used is the standard deviation. However, as with the position above, a more robust technique is required in general.

We denote the measured width of the distribution as $\sigma_{j}$. This will approach the limiting value $\sigma_{\mathrm{S}}$ of the seeing function $S_{\lambda}(x)$ as either (i) the separation of the two objects tends to zero; or (ii) the ratio of the less bright object flux to the brighter object flux tends to zero. If either of the objects becomes brighter/dimmer, then the observed width will change. The width $\sigma_{j}$ will also change with the separation of the objects. If the separation of the objects is larger than the intrinsic $\sigma_{\mathrm{S}}$ of the seeing, then the two objects will start to be resolved, and the spectroastrometric technique is not required to separate the objects' spectra. Hence the width $\sigma_{j}$ is a function of (i) the separation of the two objects; (ii) the intrinsic distribution's $\sigma_{\mathrm{S}}$; and (iii) the flux ratio of the two objects.

A slight broadening of the convolved profile, i.e. $\sigma_{j}>\sigma_{\mathrm{S}}$, is present even in the continuum, as both stars still contribute to the flux distribution. This is important in that the measured $\sigma_{j}$ of the continuum cannot be used as an estimate for $\sigma_{\mathrm{S}}$. A separate measurement for $\sigma_{\mathrm{S}}$ will be necessary (by observing a single star), particularly as the focus of the camera can change along the $\mathrm{CCD}$ producing a varying $\sigma_{\mathrm{S}}$ in the dispersion direction. It may be possible to use the minimum of $\sigma_{j}$ as an estimate for $\sigma_{\mathrm{S}}$ where there is a particularly strong emission line present from one of the objects such that $f_{1, j} \gg f_{2, j}$, although this will still be a slight overestimate for $\sigma_{\mathrm{S}}$ (see Appendix A.2 for an evaluation of the errors associated with this procedure).

In order to understand the behaviour of the changes in width of the flux distribution $\sigma_{j}$, with flux ratio, binary object separation and intrinsic width $\sigma_{\mathrm{S}}$, we have performed an extensive series of numerical simulations. The flux ratio ranged from $f_{2, j} / f_{1, j}=0.0-1.0$ (stepsize 0.05 ); the separation ranged from $d=0-3 \sigma_{\mathrm{S}}$ (stepsize $0.1 \sigma_{\mathrm{S}}$ ), and we have varied the intrinsic width $\sigma_{\mathrm{S}}$ from 2-10 pixels (similar to typical observations). Each realisation first convolves the binary object flux distribution with the function $S_{\lambda}(x)$, and then maps the resultant flux onto pixels. We have also performed the calculation for different functions $S_{\lambda}(x)$, including a Gaussian, a Gaussian with boosted wings (the Gaussian function multiplied by $\left.1+0.05\left(x / \sigma_{\mathrm{S}}\right)^{4}\right)$, top-hat and triangular functions. In all cases we have measured the width of the resultant distribution using Andrew's sine estimator, although identical results are obtained with a bi-weight estimator, or a standard-deviation width.

The results of some of these calculations are displayed in Fig. 1. We find that the behaviour of the fractional change of the width of the distribution is almost independent of the function $S_{\lambda}(x)$ and may be approximated by a fitting function. For objects with separations less than twice the intrinsic $\sigma_{\mathrm{S}}$ (i.e. $\left.d<2 \sigma_{\mathrm{S}}\right)$ an excellent fit to the calculations is

$\frac{\sigma_{j}-\sigma_{\mathrm{S}}}{\sigma_{\mathrm{S}}}=a\left(\frac{d}{\sigma_{\mathrm{S}}}\right)^{b}\left[1-\left|1-\min \left\{\left(\frac{f_{2, j}}{f_{1, j}}\right),\left(\frac{f_{1, j}}{f_{2, j}}\right)\right\}\right|^{c}\right]$.

The best fitting constants $a, b$, and $c$ (with a search stepsize of 0.001 ) in this expression are found to be slightly dependent on the exact shape of the convolving function $S_{\lambda}(x)$. The best fitting values are listed in Table 1.

This expression is shown in Fig. 1 for different functions $S_{\lambda}(x)$. Equation (5) reproduces the numerical results within a few per cent of the calculated value for all of the convolution functions $S_{\lambda}(x)$ we have used (although it is slightly worse for the triangular function than the other functions). The error associated with use of this fit produces a systematic shift in the separated fluxes (especially for the dimmer secondary object) in the practical application of the technique (see Sect. 3). To reduce this error, we can actually measure the function $S_{\lambda}(x)$, again via a single object observation, and then use it to calculate either more accurate values of $a, b$, and $c$ in Eq. (5), or a "lookup" table of values for use in flux separation. Equation (5) is important in that this representation of the 

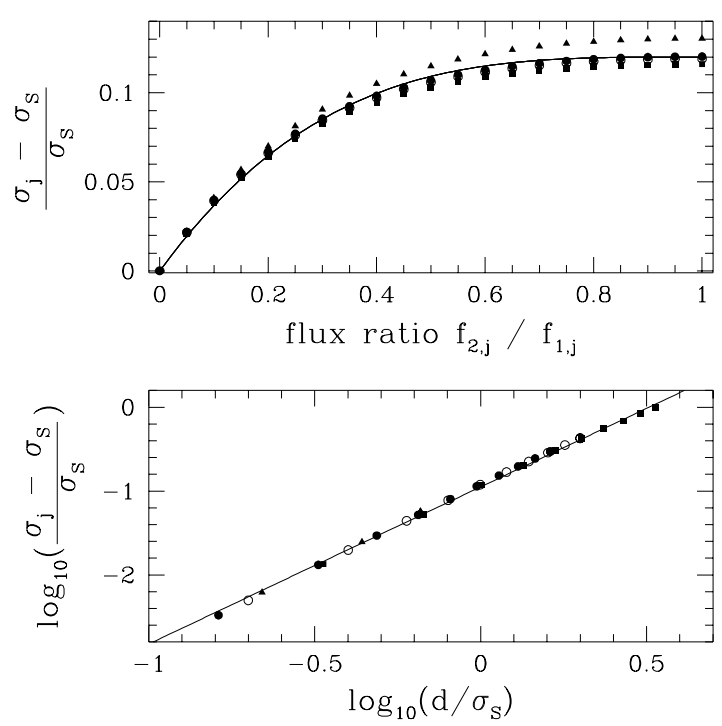

Fig. 1. Top panel: variation of the width of the distribution with flux ratio. The symbols are the calculated data for an intrinsic convolving function of a Gaussian (open circles, width 5.0 pixels), a Gaussian with boosted wings (filled circles, width 6.2 pixels, see text) a top-hat (squares, width 3.0 pixel), and a triangle (triangles, width 4.6 pixels). The separation between the sources is 3.0 pixels. All results have been divided by $\left(d / \sigma_{\mathrm{S}}\right)^{b}$ ( $b$ from Eq. (5) listed in Table 1) to show the dependency of the width on the flux ratio only. Bottom panel: variation of the width with separation $d$ between the sources for a flux ratio of $f_{2, j} / f_{1, j}=0.5$. Symbols are the same as for the top panel. The solid lines in both panels are the fit of Eq. (5).

Table 1. Best fitting parameters to Eq. (5), $S_{\lambda}(x)$ is the convolving function - see text.

\begin{tabular}{lrrr}
\hline \hline$S_{\lambda}(x)$ & $a$ & $b$ & $c$ \\
\hline Gaussian & 0.116 & 1.930 & 3.468 \\
Gaussian with boosted wings & 0.118 & 1.951 & 3.468 \\
Top hat & 0.110 & 1.858 & 3.466 \\
Triangular & 0.130 & 2.012 & 3.448 \\
\hline
\end{tabular}

change in the width contains all of the convolution information of the binary flux distribution.

\section{Method of extraction of the individual spectra}

The total flux $f_{\text {tot, } j}$ is dependent on the fluxes of the two point sources $f_{1, j}$ and $f_{2, j}$. The position displacement $\mu_{j}^{\prime}$ is dependent on $f_{1, j}, f_{2, j}$ and the separation $d$. Finally, the width of the flux distribution $\sigma_{j}$ is dependent on $f_{1, j}, f_{2, j}$ and $d$. Therefore measurement of (i) the total flux $f_{\text {tot }, j}$; (ii) the position displacement $\mu_{j}^{\prime}$; and (iii) the width of the flux distributed in the spatial direction $\sigma_{j}$ provides three observables for three unknowns (i.e. the fluxes of the two objects $f_{1, j}, f_{2, j}$, and their separation $d$ ). The set of three relations may be inverted to uniquely separate the flux distribution into its components.

Previous attempts at spectroastrometric flux separation have used the total flux $f_{\text {tot }, j}$ and position displacement $\mu_{j}^{\prime}$ as their only two observables. To be successful, prior knowledge of the objects must be obtained (e.g. Bailey 1998a knew the separation $d$ of the binary). If this is not possible, then we have three unknowns (the two fluxes and source separation) and only the two observables. Hence flux separation may not be uniquely achieved as we do not have a closed set: attempts to separate the flux using only two observables may produce misleading results.

We use the following method to deconvolve the spectra: first for a given value of the separation $d$, we calculate the flux ratio in the continuum $r_{\text {cont }}$ with Eq. (5) and the observed width $\sigma_{\text {cont }}$. Then for each row in the dispersion direction $j$ we invert Eq. (4) using the observed centroid $\mu_{j}^{\prime}$ and continuum ratio $r_{\text {cont }}$ to produce the flux ratio $r_{j}$. Then with $f_{\text {tot }, j}=f_{1, j}+f_{2, j}$, we calculate the individual spectra $f_{1, j}$ and $f_{2, j}$. Using these values, we predict the width of the distribution using Eq. (5), and compare to the observed values using a simple $\chi^{2}$ calculation to evaluate the fit. Finally we repeat this procedure with differing values of the separation $d$ until a best fit is found.

We follow this procedure for two examples (see below). This suggested method applies in the case that the width $\sigma_{\mathrm{S}}$ of the convolving function is known ${ }^{1}$. The separation $d$ is the independent parameter which is varied to minimise $\chi^{2}$ for the fit.

\subsection{Example $A$}

In order to fully test the method, a series of emission and absorption "lines" of differing contrasts and widths are imposed on the continuum of both objects (see the top two panels in Fig. 2). These provide parts of the spectrum which have all combinations of the primary and secondary object brightening and dimming over the lines: from the left (in increasing pixels) the features correspond to (i) object 1 emission line only; (ii) object 2 absorption only; (iii) large emission line in object 2 such that it is brighter than object 1; (iv) both objects absorption; (v) object 1 emission, object 2 absorption; (vi) both objects emission; (vii) object 1 absorption, object 2 emission; (viii) both objects absorption, but with the absorption minima offset; (ix) both objects emission with identical contrast; and finally (x) both objects with absorption lines of identical contrast. Note that the two fluxes are not meant to represent any sort of object or binary system in particular: they are simply meant to illustrate the differing combinations of primary and secondary flux. The continuum flux ratio is constant at all wavelengths at $r_{\text {cont }}=0.2$.

An "observation" is then made: this consists of convolving the input with $S_{\lambda}(x)$ assumed to be a Gaussian with a width (identical to the standard deviation in this case) of $\sigma_{\mathrm{S}}=$ 4.0 pixels. The resultant distribution is then binned into pixels in position and dispersion. The observables are then calculated - the total flux, the width, and position offset. To derive the relative change in width, one of the input fluxes is set to zero, and an "observation" taken of a single flux component, and the width calculated. The derived observables for a source separation of 2.0 pixels are displayed in the third-fifth panels of Fig. 2 for a range of signal-to-noise ratios $(S N R)$.

1 We note that this procedure may be changed if the prior information is varied: if the separation is accurately known then a similar method could be devised which had the width $\sigma_{\mathrm{S}}$ as the independent parameter. 


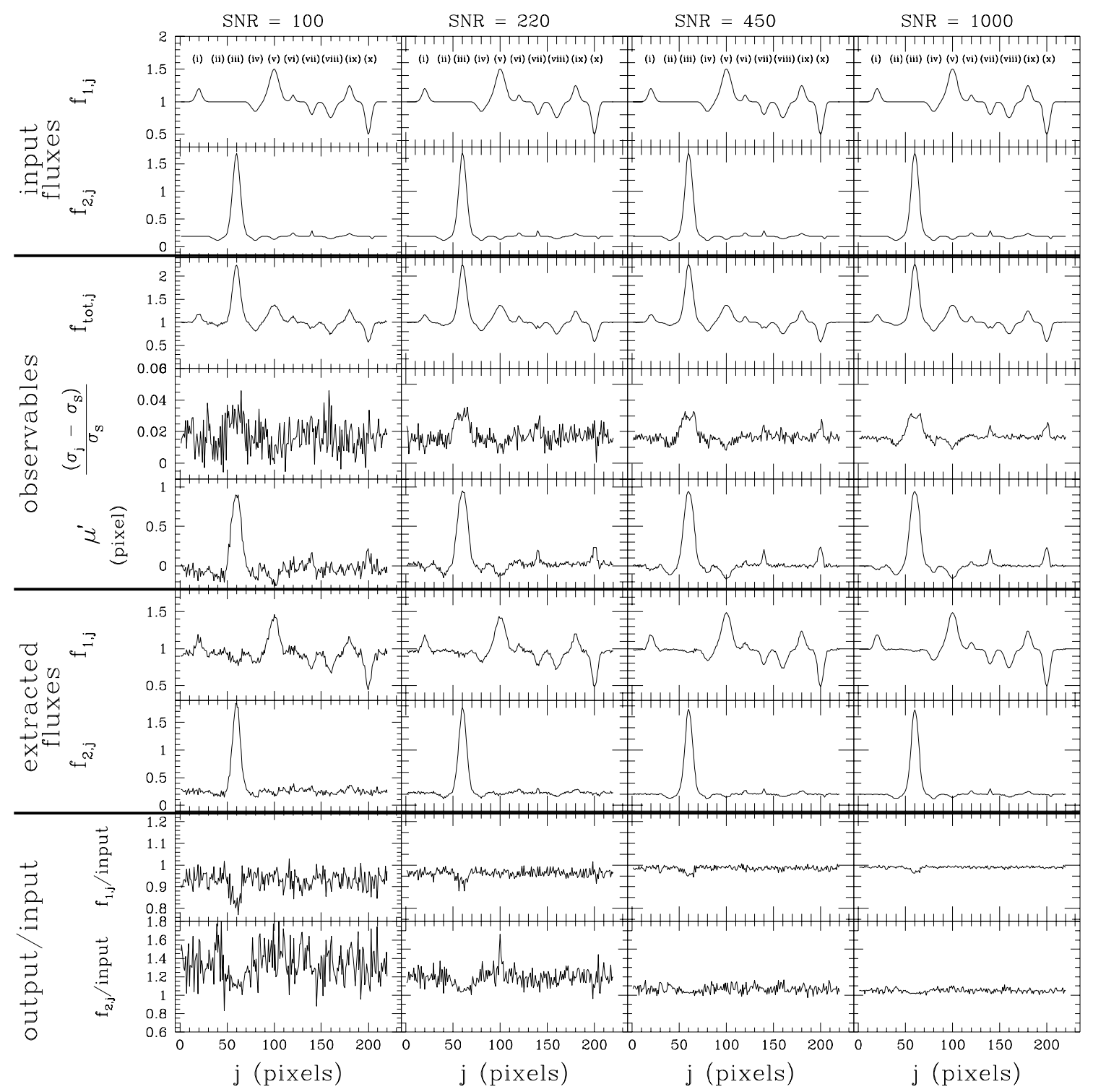

Fig. 2. Example A: the top two panels are the input fluxes of the individual objects (the primary object's flux is normalised to unity in the continuum). The next three panels are the observable quantities: the total flux, the relative change in width of the distribution, and the change in position centroid. The next two panels are the best-fit extractions of the two fluxes, and the bottom two panels are the ratio of the extracted flux to the input fluxes from the top two panels. The separation of the binary is $d=2.0$ pixels, and the convolving function is Gaussian with width $\sigma_{\mathrm{S}}=4.0$ pixels.

One feature in the width distribution is particularly worthy of note. When the fainter object in the continuum has a large emission line - feature (iii) in Fig. 2 - the width of the flux distribution $\sigma_{j}$ can be seen to decrease in the centre of the line, producing a double-peaked profile. The distribution width $\sigma_{j}$ has a maximum for a flux ratio of unity. How does the width $\sigma_{j}$ change from the line wing of feature (iii) to line centre? In the wing, object 2 is much less bright than object 1, and the flux ratio in Eq. (5) is less than unity. As the line emission increases, the flux ratio increases (and hence so does the width $\sigma_{j}$ ) until the emission from both objects is equal and the flux ratio is unity. Here, the width of the distribution $\sigma_{j}$ reaches its maximum value. As the line emission increases further such that object 2 is brighter than object 1 , the width $\sigma_{j}$ decreases because $\sigma_{j}$ is dependent on the minimum of the ratios $r_{j}$ and $1 / r_{j}$ (this is because there must be no difference in $\sigma_{j}$ whichever of the two objects is denoted object 1).

This produces a maximum in the width $\sigma_{j}$ which is offset in wavelength from the maximum in emission, and hence a double peaked profile in $\sigma_{j}$ is observed. The peaks in $\sigma_{j}$ then correspond to a flux ratio of unity.

The bottom four panels of Fig. 2 show the results of the separation method. Panels six and seven display the extracted fluxes, and panels eight and nine show the extracted fluxes divided by the input fluxes (if the extraction is perfect, then these panels should be unity). It is clear from examination of these four panels that the flux separation method achieves good results even for $S N R$ of 100 , and that as $S N R$ increases, the accuracy of the flux separation becomes more accurate. To find the best-fit, the distance between the components $d$ was 


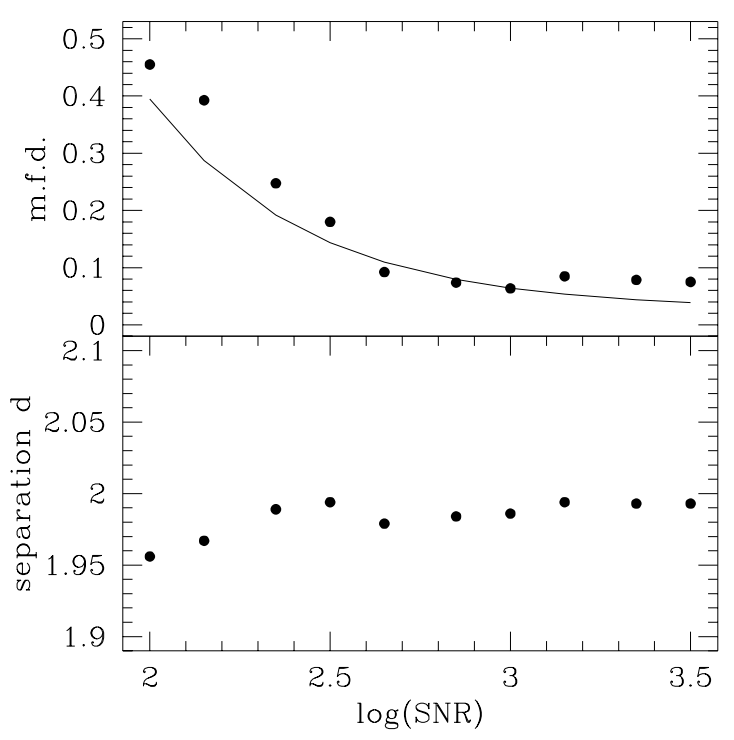

Fig. 3. Variation of mean fractional deviation per pixel of the extracted fluxes from the input fluxes (Eq. (6)) (top panel), and best-fit separation (bottom panel) for Example A with increasing signal-to-noise ratio $(S N R)$. The solid line in the top panel is the mean fractional deviation per pixel expected for this example, with systematic error of $1 \%$ see Sect. 4.

varied from $0-5$ pixels with a stepsize of 0.001 pixels. This procedure yielded $d=1.956,1.989,1.979$ and 1.986 pixels for SNR of 100, 220, 450, and 1000 respectively.

To assess how well the flux separation was achieved we calculate a mean fractional deviation per pixel of the extracted flux:

m.f.d. $=\frac{1}{m} \sum_{j=0}^{j=m}\left[\left|\frac{f_{1, j, \mathrm{ex}}}{f_{1, j, \mathrm{in}}}-1\right|+\left|\frac{f_{2, j, \mathrm{ex}}}{f_{2, j, \mathrm{in}}}-1\right|\right]$

where the subscript "ex" and "in" refer to the extracted and input fluxes respectively. The m.f.d. per pixel for Example A along with the best-fit separation $d$ as a function of SNR are shown in Fig. 3. The separation asymptotes to the input separation of 2.0 pixels, and the m.f.d. per pixel decreases with increasing $S N R$. However, even for $S N R>1000$, the separation is not exact, and indeed, is not expected - this is an empirical method, and hence will contain errors (see Sect. 4 below). The solid line on the top panel of Fig. 3 is the expected m.f.d. per pixel for this simulation as described below in Sect. 4.

Figures 2 and 3 illustrate the performance of the method as the $S N R$ varies for a constant continuum flux ratio. We also investigate the method for differing continuum flux ratios $r_{\text {cont }}$ and distance between the objects $d$. For this we have conducted a series of simulations for the same features in the spectra (top two panels in Fig. 2). However, the continuum flux ratio $r_{\text {cont }}=$ $f_{2 \text {,cont }} / f_{1 \text {,cont }}$ is varied from unity to $1 / 50$, and the separation $d$ of the sources ranges from 0.1 pixels to 4.0 pixels (i.e. $0.025-1.0$ of the standard deviation of the Gaussian convolving function). For all of these simulations, the noise level is fixed to produce $S N R=220$ (similar to that in the simulations of Courbin et al. 2000 for comparison of the two methods).

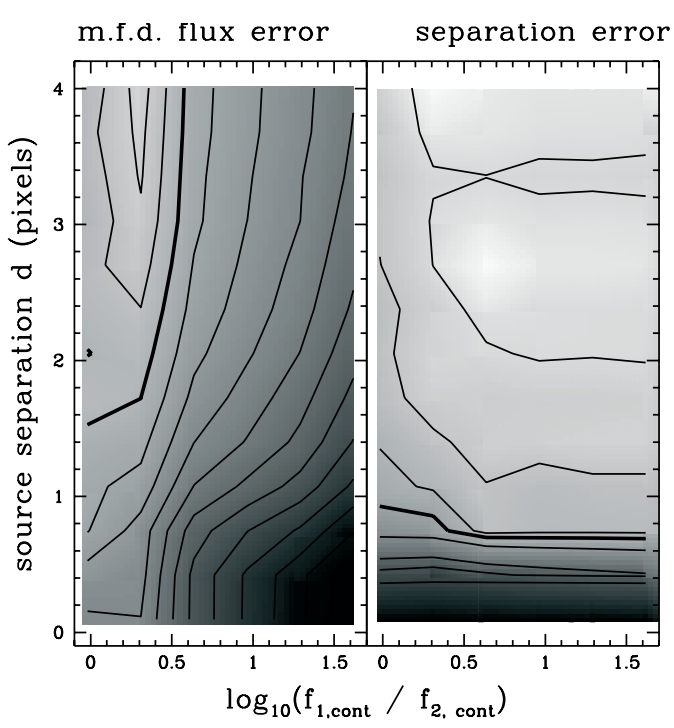

Fig. 4. Map of mean fractional deviation per pixel (left) and error in the best-fit separation (right) for a set of simulations using the input spectra of Example A, but varying the flux ratios and the object separation. The contours are logarithmically spaced with steps of 0.2 dex. The bold contours correspond to an error of 0.1 (i.e. 10\%). In the left panel, the highest contour corresponds to an $4.0 \%$ error and is situated in the top left corner. In the right panel the highest contour corresponds to $2.6 \%$ and is in the middle-top right of the panel.

For each pair of $r_{\text {cont }}$ and $d$ we calculate the m.f.d. per pixel of the extracted fluxes and plot this as a contour map in the left panel of Fig. 4. The contours are spaced logarithmically in intervals of 0.2 , and the bold contour marks the m.f.d. per pixel of $0.1(\log ($ m.f.d. $)=-1)$. We can clearly see that the technique performs best for large separations $d$ and for flux ratios $r_{\text {cont }}$ close to unity. We also note that accurate extraction of the fluxes can be achieved for object separations of less than one pixel (a feature of the technique which, to our knowledge, is unique). We have also calculated the fractional error in the bestfit separation and show the contour map of this in the right hand panel of Fig. 4. Again the contours are spaced in 0.2 dex, and the bold line corresponds to a fractional error of 0.1 (i.e. 10\%). The main feature of this map is that the best-fit separation $d$ is not very sensitive to the continuum flux ratio, and that accuracy of better than $10 \%$ should be possible for input separations of larger than around one pixel.

\subsection{Example $B$}

To provide a direct comparison with previous methods, we adopt exactly the same problem considered by Courbin et al. (2000) for a second test. This consists of a quasar and a star with a featureless continuum (see Courbin et al. 2000, Sect. 3.1). The quasar spectrum used is the mean of all objects taken from the $2 \mathrm{QZ}$ project $^{2}$ (Croom et al. 2004). The two point sources are separated by 2.0 pixels, and the convolving Gaussian to provide the seeing has a width of 4.0 pixels. The relative continuum brightness ranges from $r_{\text {cont }}=f_{2, \text { cont }} / f_{1 \text {,cont }}=0.2-1$ from the blue to the red end of the

\footnotetext{
${ }^{2}$ http://www.2dfquasar .org/
} 


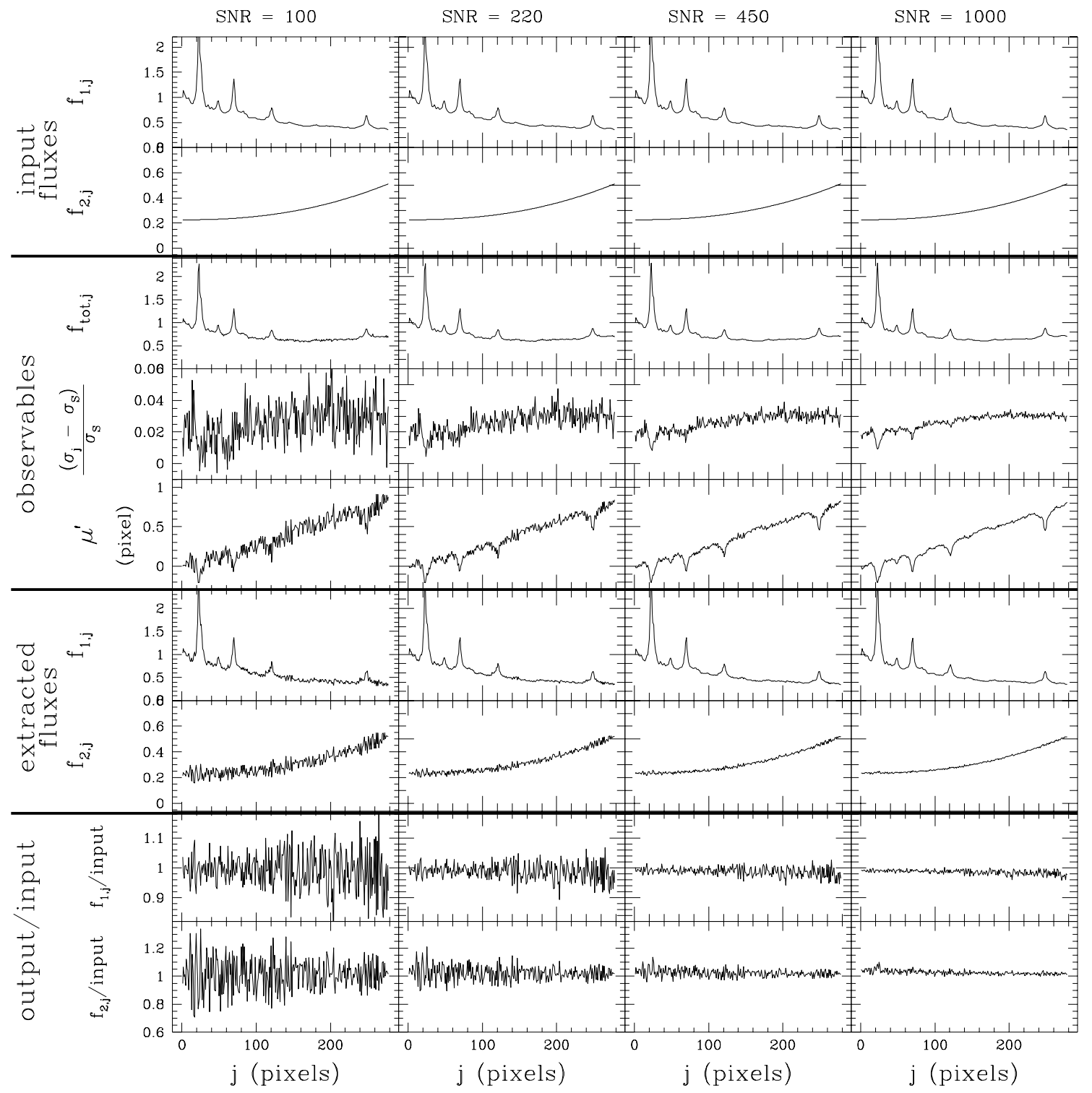

Fig. 5. Example B: the top two panels are the input fluxes of the individual objects. The next three panels are the observable quantities: the total flux, the relative change in width of the distribution, and the change in position centroid. The next two panels are the best-fit extractions of the two fluxes, and the bottom two panels are the ratio of the extracted flux to the input fluxes from the top two panels. The separation of the binary is $d=2.0$ pixels, and the convolving function is Gaussian with width $\sigma_{\mathrm{S}}=4.0$ pixels.

spectra. The mean flux ratio of the two components is larger in this case than in Example A, and therefore we should expect more accurate results.

Figure 5 shows the results for this test in a similar fashion as Fig. 2, and Fig. 6 shows how the m.f.d. per pixel in the extracted fluxes and the best-fit separation vary with $S N R$. As can be seen, the extraction does indeed produce more accurate results than for Example A. For the cases of $S N R=200-300$ (exactly the same as in Courbin et al.) the m.f.d. per pixel is $\approx 0.05$. We estimate that this is larger than the typical m.f.d. in the results of Courbin et al. (from the inserts in their Fig. 3) by a factor of $\sim 2$. This indicates that the spectroastrometric method of extracting fluxes is not quite as efficient as Courbin et al.'s method for this particular example - unfortunately Courbin et al. do not discuss differing $S N R$ simulations.
As in Example A, we examine the technique for differing values of continuum ratios and component separations (the continuum ratio is defined by the fluxes at pixel $j=1$ ). Figure 7 displays the contour map of m.f.d. per pixel and best-fit separation (as Fig. 4 for Example A), for variation of the continuum ratio (unity $-1 / 50$ ) and component separation $(d=$ $0.1-4.0$ pixels). We have fixed the $S N R$ at 220. Again, the most accurate results are obtained for larger component separations $d$, and for larger flux ratios.

\section{Random and systematic errors}

We can calculate the expected fractional deviation per pixel in a straightforward way. At each pixel $j$, small variations in the fluxes $f_{1, j}$ and $f_{2, j}\left(\delta f_{1, j}\right.$ and $\delta f_{2, j}$ respectively) produce a variation in the flux ratio $\delta r_{j}$, where $\delta r_{j} / r_{j}=\delta f_{2, j} / f_{2, j}-\delta f_{1, j} / f_{1, j}$. 


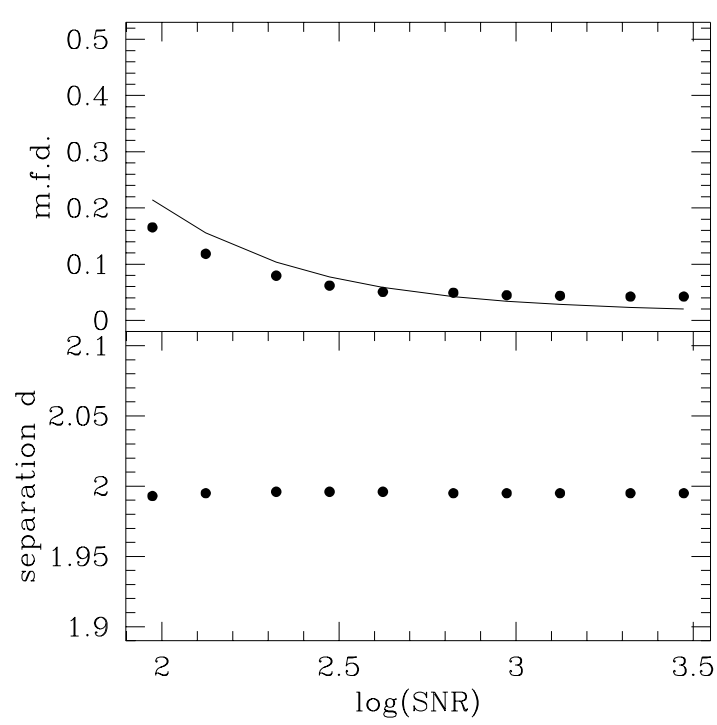

Fig. 6. Variation of mean fractional deviation per pixel of the extracted fluxes from the input fluxes (Eq. (6)) (top panel), and best-fit separation (bottom panel) for Example B with increasing signal-to-noise ratio $(S N R)$. The solid line in the top panel is the m.f.d. per pixel expected for this example, with systematic error of $1 \%$ see Sect. 4.

As the sum of the two fluxes is a constant for any $j$, then the small variations $\delta f_{1, j}$ and $\delta f_{2, j}$ are equal and opposite in sign $\left(\delta f_{1, j}=-\delta f_{2, j}\right)$. Hence, $\delta r_{j} / r_{j}=-\left(1+1 / r_{j}\right) \delta f_{1, j} / f_{1, j}$.

The mean fractional deviation per pixel (as defined in Eq. (6)) is the sum of the absolute fractional errors:

m.f.d. $=\frac{\left|\delta f_{1, j}\right|}{f_{1, j}}+\frac{\left|\delta f_{2, j}\right|}{f_{2, j}}=\frac{\left|\delta f_{1, j}\right|}{f_{1, j}}\left(1+\frac{1}{r_{j}}\right)=\frac{\left|\delta r_{j}\right|}{r_{j}}$.

In the limit that the flux ratio $r_{j} \ll 1$, the expected error in $r_{j}$ is calculated in Appendix A.1 to be

$\left|\delta r_{j}\right| \approx \frac{1}{a c \sqrt{2} S N R}\left(\frac{\sigma_{\mathrm{S}}}{d}\right)^{b}+\left.\delta\right|_{\mathrm{syst}}$

where $a, b$, and $c$ are the constants in Eq. (5), and $\left.\delta r\right|_{\text {syst }}$ is the systematic error from the fitting formula (5)

We can use this estimate to calculate the expected m.f.d. error for Examples A and B. Inserting a systematic error of $\left.\delta r\right|_{\text {syst }}=0.01$ (i.e. $1 \%$ ), the resultant predictions for the m.f.d. per pixel are plotted as the solid line on the top panels of Figs. 3 and 6. We note that, in deriving the expressions above, we have assumed that $r_{j} \ll 1$, and so our calculations are not directly applicable to either Examples A and B, which have mean flux ratios per pixel of 0.18 and 0.36 . Therefore we expect a closer fit to the numerical results for Example A than for Example B, which is seen in Figs. 3 and 6. The predicted m.f.d. per pixel are good estimates for the calculated values.

\section{Observing practicalities}

The method is clearly able to separate binary object fluxes if the $S N R$ is high enough. However, there are several practical aspects of the technique which need to be noted. Whilst some of these are discussed in detail in Bailey (1998a), and also in Baines (2004) it is useful to provide a list including aspects gleaned in the development of the method.

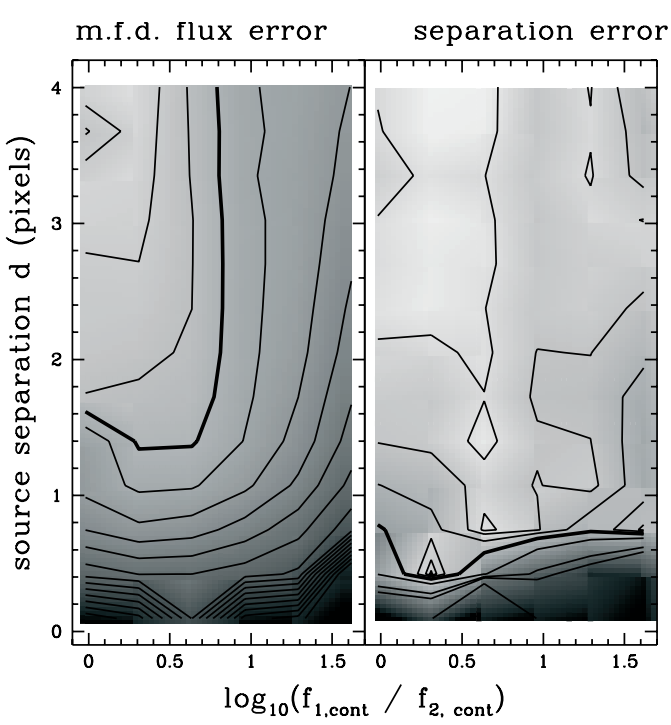

Fig. 7. Map of mean fractional deviation (left) and error in the best-fit separation (right) for a set of simulations using the input spectra of Example B, but varying the flux ratios and the object separation. The contours are logarithmically spaced with steps of 0.2 dex. The bold contours correspond to an error of 0.1 (i.e. 10\%). In the left panel, the highest contour corresponds to an $2.6 \%$ error and is situated in the top left corner. In the right panel the highest contour corresponds to $2.6 \%$ and is in the middle-top left of the panel.

1. Both components of the binary must be fully in the spectrograph slit else unknown fractions of the flux from one component will be observed, and so the method will fail. Use of a wide slit will degrade the spectral resolution of the final observation a little, although this is a small sacrifice as it is of paramount importance that both objects are fully within the slit. If it is not known a priori whether the object is a binary or not, then a wide slit should be used.

2. Full sampling of the convolved flux distribution is essential - as already stated at least 4 pixels (or equivalent for dithering patterns) must sample this function to avoid Nyquist sampling problems.

3. For some spectrographs, it is easily possible to repeat the observation with the instrument rotated through $180^{\circ}$. This is useful as it allows any instrumental misalignment problems to be subtracted - this point is particularly stressed in Bailey (1998a) and is worth repeating here.

4. The flux separation method will only operate in the spatial direction parallel to the slit axis. If the line-of-centres axis is orientated normal to the slit axis, then no flux separation will be possible. At intermediate angles between these two axes then the best-fit separation will be equal to the actual separation multiplied by the cosine of the angle between the axes. Information on the position angle of the distribution may be obtained by combining measurements of two observations taken with the spectrometer slit at two different angles on the sky (e.g. see Baines et al. 2004).

5. An important aspect of the method is to observe a single object with a similar position on the sky as the intended target in order to measure the intrinsic width of the convolution 
function. These single object observations can also be used to calculate series of convolutions of binary flux distributions, in order to generate a "look-up" table of values to be used in the deconvolution, instead of using Eq. (5). In practice, this may be difficult: the seeing can change with time, making single star observations less useful. When one object has a particularly strong emission line, then the measured width of the flux distribution at line centre $\sigma_{j}$ may be used as an estimator for the intrinsic width $\sigma_{\mathrm{S}}$ (see Appendix A.2).

\section{Discussion}

Given the volume of published work dedicated to the deconvolution of images, why are we investigating new techniques to achieve the same result? As the image is sampled in discrete intervals (the pixels), then any deconvolution method can only increase its resolution (a result from sampling theory). The fundamental aspect of the spectroastrometric technique is that it utilises changes in the flux distribution convolved with the PSF. As this convolution takes place before the distribution is sampled onto the pixels, then information on scales smaller than the pixels can be retrieved (this is a different approach to the problem than deconvolution methods, such as those described in Courbin et al. 2000). This is because the information about the binary is spread throughout the PSF. Therefore, if the PSF itself is well sampled then extraction of point sources separated by less than the traditional spatial Nyquist sampling interval (pixel) is possible. Hence extraction of fluxes is possible at a much higher spatial resolution than with previous methods. Of course, there is a price to pay for this advantage over deconvolution techniques. We have assumed a priori two point sources, and so have imposed a criterion on the flux distribution (a feature not present in traditional deconvolution methods). Also this is essentially an empirical method - method noise will always be present in the solution.

Spectroastrometry is likely to achieve best results for the cases when specific known binary sources are targeted. Serendipitous binary discovery may prove frustrating when it is clear from the data that a binary component is present, although little else may be derived because, for example, the secondary component is not fully in the slit, or that the $S N R$ is not high enough to extract the individual fluxes reliably. The technique is sufficiently general that any binary object may be examined (although triple systems may cause problems!).

How powerful is the technique? For ground based telescopes, the dominant contributor to the point spread function is the seeing, which typically ranges from one-few arcsec. With an equivalent signal to noise ratio of several hundred (often achieved with modern instruments) and pixel sizes of a few tenths of an arcsec (typical of spectrographs), the position centroid may be determined to $\sim$ milliarcsecond accuracy. Spectroastrometry should then be a natural complementary technique to the growing optical/IR interferometry field which operates at a similar angular scale.

Future investigations into spectroastrometry will concentrate on continuous flux distributions, such as discs, where the main goal will be to measure the kinematics of the emitting gas on sub-pixel scales. This technique is difficult, in that high SNRs are necessary (and hence the observational aspects may be challenging), but does promise great rewards.

\section{Conclusion}

We describe the technique of spectroastrometry and present a method to separate individual binary object fluxes from the point-spread function which is present in all observations. The method makes use of the total flux, the position offsets and the characteristic width of the flux distribution in the spatial direction: the three observational quantities it is possible to measure directly from a long slit spectrograph image. The observing technique to achieve this is not difficult for careful observers.

We have demonstrated that the performance of the technique is similar to previously published deconvolution methods which reconstructs the original flux distribution on the sampling scale ( pixels). Although spectroastrometry cannot achieve this complete mapping, it is the only technique in our knowledge, which is able to successfully separate the fluxes of binary objects with sub-pixel separations.

The technique of spectroastrometry is a natural counterpart to interferometry, as it provides spectral information (and hence kinematics) at similar resolutions, which with available instrumentation is typically milliarcseconds.

Acknowledgements. We thanks the anonymous referee for very helpful comments on the submitted version of this paper. D.B. thanks PPARC for postgraduate studentship award.

\section{Appendix A: Random and systematic errors}

If the width $\sigma_{j}$ of the spatial distribution of the flux is sampled over at least two pixels (so the profile will extend over at least 4 pixels), then the extent of the profile does not effect the error estimates (essentially this is an expression of Nyquist sampling).

The two error sources will be from photon counting statistics and read-noise. Where the read-noise errors are dominant in determining the pixel signal-to-noise ratio $(S N R)$, it can be justified that as long as the errors are symmetrically distributed around zero then their net effect on the $\mu_{j}$ and $\sigma_{j}$ should be zero (especially when robust location and width estimators are used).

\section{A.1. Error in the flux ratio}

When photon noise dominates, the error in the number of photons $F_{i j}$ in each pixel is simply $\delta F_{i j}=F_{i j}^{1 / 2}$. The signal-to-noise ratio of the position-integrated flux is $S N R_{j}=$ $\left(\sum_{i} F_{i j}\right) /\left(\sum_{i} F_{i j}\right)^{1 / 2}=\left(\sum_{i} F_{i j}\right)^{1 / 2}$. The standard errors in the position centroid and width (mean and standard deviation) are the familiar expressions $\delta \mu_{j}^{\prime}=\sigma_{j} / n_{j}^{1 / 2}$ and $\delta \sigma_{j}=\sigma_{j} /\left(2 n_{j}\right)^{1 / 2}$, where $n_{j}$ is the number of counts in the position-integrated profile (e.g. Topping 1972). Assuming that the $S N R$ is the same 
across the spectrum (i.e. $S N R=S N R_{j}$, identical for all $j$ ) we find

$\delta \mu_{j}^{\prime}=\frac{\sigma_{j}}{S N R}$ and $\delta \sigma_{j}=\frac{\sigma_{j}}{\sqrt{2} S N R}$.

The more the seeing function deviates from a Gaussian, then these expressions for the errors $\delta \sigma_{j}$ and $\delta \mu_{j}^{\prime}$ become less accurate (e.g. see Beers et al. 1990).

For a given error in the position centroid and width we can estimate the typical error in using the technique. Let us assume that the random error in the intrinsic width of the seeing profile is small in comparison with $\delta \sigma_{j}$, i.e. $\delta \sigma_{\mathrm{S}} \ll \delta \sigma_{j}$ (see A.2 for the consequences of incorrectly deriving $\sigma_{\mathrm{S}}$ ). Furthermore, let us assume that the random error in the separation $\delta d$ is small, and so the dominant error source in the flux ratio is via the variation in the width $\sigma_{j}$ (our method of solution uses the separation $d$ as the independent variable to minimise the $\chi^{2}$, and hence $\delta d$ is dominated by systematic errors).

Rearrangement of Eq. (5) to make the flux ratio $r_{j}=$ $f_{2, j} / f_{1, j}$ the subject allows a simple error analysis to be performed. This produces the random error in the flux ratio $\delta r_{j}$ of:

$\left.\delta r_{j}\right|_{\mathrm{rand}} \approx \frac{1}{a c\left(\frac{d}{\sigma_{\mathrm{S}}}\right)^{b}}\left(\frac{\delta \sigma_{j}}{\sigma_{\mathrm{S}}}\right)$,

where we have also assumed that $r_{j} \ll 1$, i.e. the case for small flux ratios. There will also be a systematic error in this ratio $\left.\delta r\right|_{\text {syst }}$ related to the inaccuracy in the fitting formula (5), leading to the total error in the flux ratio as

$$
\begin{aligned}
\delta r_{j} & =\left.\delta r_{j}\right|_{\mathrm{rand}}+\left.\delta r_{j}\right|_{\mathrm{syst}} \\
& \approx \frac{1}{a c}\left(\frac{\sigma_{\mathrm{S}}}{d}\right)^{b}\left(\frac{\delta \sigma_{j}}{\sigma_{\mathrm{S}}}\right)+\left.\delta r\right|_{\mathrm{syst}} \\
& \approx \frac{1}{\sqrt{2} a c}\left(\frac{\sigma_{\mathrm{S}}}{d}\right)^{b}\left(\frac{1}{S N R}\right)+\left.\delta r\right|_{\mathrm{syst}}
\end{aligned}
$$

where, for the final expression, we have inserted Eq. (A.1).

\section{A.2. Systematic errors in the intrinsic $\sigma_{S}$ and its time variation}

We have assumed that the measurement error in the intrinsic width of the flux distribution $\sigma_{\mathrm{S}}$ is zero. In practice this may not be the case as there is no high $S N R$ observation of a single object or where the seeing is changing over time. An estimate of $\sigma_{\mathrm{S}}$ could be made from the binary objects' observations by assuming that the smallest measured value of $\sigma_{j}$ occurs when one of the objects completely dominates the output: this is correct in the limit $f_{2, j} / f_{1, j} \rightarrow 0$, as then $\sigma_{j} \rightarrow \sigma_{\mathrm{S}}$. If this approximation is used, then $\sigma_{\mathrm{S}}$ will in general be slightly overestimated. What are the consequences from this assumption?

Both the best-fit separation between the components, and the individual fluxes (notably the continuum flux ratio) will have an associated error. From examination of Eq. (5) we see that the fractional change in the separation caused solely by an change in $\sigma_{\mathrm{S}}\left(\delta \sigma_{\mathrm{S}}\right)$ is

$\frac{\delta d}{d}=\frac{\delta \sigma_{\mathrm{S}}}{\sigma_{\mathrm{S}}}\left[-\frac{1}{b}\left(\frac{\sigma_{\mathrm{S}}}{\sigma_{j}-\sigma_{\mathrm{S}}}\right)+1-\frac{1}{b}\right]$

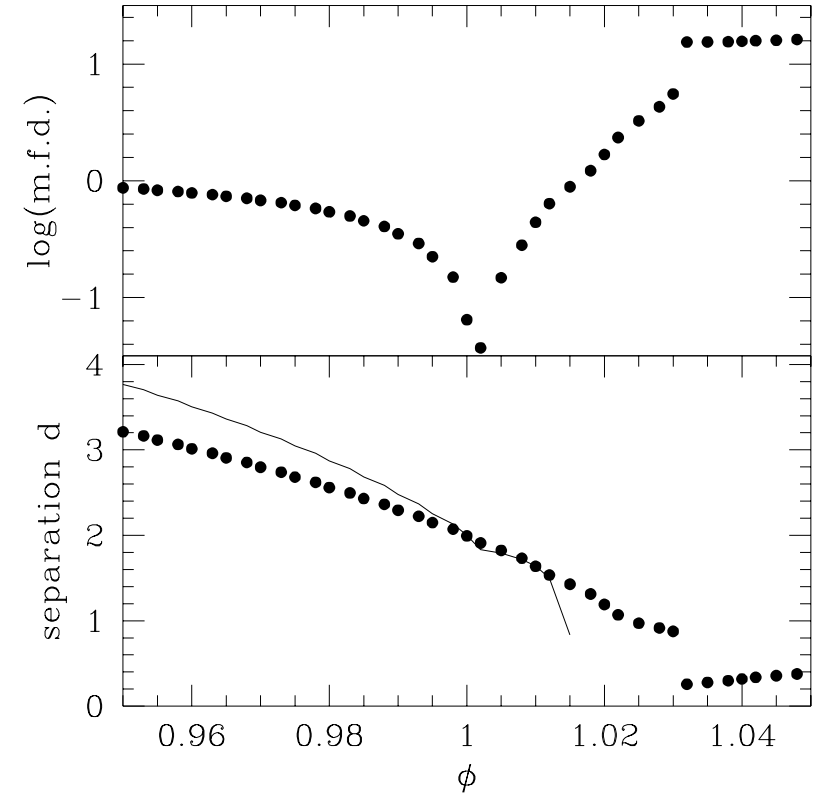

Fig. A.1. Top panel: logarithm of the m.f.d. per pixel versus the systematic error in the intrinsic width of the seeing $\sigma_{\mathrm{S}}$. Bottom panel: the variation of the best fit separation. See text.

which assumes that there is no resultant error in the flux ratio (strictly this is never the case, but we proceed in the spirit of producing relatively simple error estimates). Note that an overestimate of $\sigma_{\mathrm{S}}$ leads to a systematic underestimate of $d$.

We have calculated a series of simulations for Example A in the text with high SNR of 3000, and systematically changed the value of $\sigma_{\mathrm{S}}$ used in the flux separation. We take the actual value of $\sigma_{\mathrm{S}}$ and multiply by a fraction $\phi$ ranging from $0.95-1.05$ to mimic both an underestimate and an overestimate of $\sigma_{\mathrm{S}}$ from the data. The results are shown as the filled circles in Fig. A.1: the top panel is the logarithm of the m.f.d. per pixel, and the bottom panel is the best-fit separation between the components. It is clear that $\phi \approx 1$ (close to unity) produces the best results by far. This underlines the desirability for accurate measurement of $\sigma_{\mathrm{s}}$. When $\phi>1.03$, the numerical method fails, as all the values of $\left(\sigma-\sigma_{\mathrm{S}}\right) / \sigma_{\mathrm{S}}<0.0$. This produces a discontinuity in the trends in Fig. A.1.

Plotted as a solid line on the bottom panel is our estimate (above, with $b=1.93$ ) for the variation in $d$. For each value of $\phi$ we calculate a mean value of $\left(\sigma-\sigma_{\mathrm{S}}\right) / \sigma$ per pixel for Eq. (A.4) (this produces the main difference between our estimated values from Eq. (A.4) and the numerical values). The calculated value predicts a zero crossing in $d$ at $\phi=1.017$, as there the mean value of $\left(\sigma-\sigma_{\mathrm{S}}\right) / \sigma_{\mathrm{S}}$ is zero.

\section{References}

Bagnuolo, W. G., Jr., Gies, D. R., \& Wiggs, M. S. 1992, ApJ, 385, 708

Bailey, J. 1998a, SPIE Proc., 3355, 932

Bailey, J. 1998b, MNRAS, 301, 161

Baines, D. 2004, Ph.D. Thesis, University of Leeds, UK 
Baines, D., Oudmaijer, R. D., Mora, A., et al. 2004, MNRAS, 353, 697

Beckers, J. 1982, Opt. Acta., 29, 361

Beers, T. C., Flynn, K., \& Gebhardt, K. 1990, AJ, 100, 32

Christy, J., Wellnitz, D., \& Currie, D. 1983, in Current Techniques in double and multiple star research, ed. R. Harrington, \& O. Franz, IAU Coll., 62, 28

Courbin, F., Magain, P., Kirkove, M., \& Sohy, S. 2000, ApJ, 529, 1136

Croom, S. M., Smith, R. J., Boyle, B. J., et al. 2004, MNRAS, 349, 1397

Garcia, P. J. V., Thiébaut, E., \& Bacon, R. 1999, A\&A, 346, 892

Goodall, C. 1983, in Understanding Robust and Exploratory Data Analysis, ed. D. C. Hoaglin, F. Mosteller, \& J. W. Tukey (New York: Wiley), 339
Hadrava, P. 1995, A\&AS, 114, 393

Lucy, L. B., \& Walsh, J. R. 2003, ApJ, 125, 2266

Magain, P., Courbin, F., \& Sohy, S. 1998, ApJ, 494, 472

Press, W. H., Teukolsky, S. A., Vetterling, W. T., \& Flannery, B. P. 1986, Numerical Recipes. The art of scientific computing (CUP)

Simon, K. P., \& Sturm, E. 1994, A\&A, 281, 286

Takami, M., Bailey, J., \& Chrysostomou, A. 2003, A\&A, 397, 675

Takami, M., Bailey, J., Gledhill, T. M., Chrysostomou, A., \& Hough, J. H. 2001, MNRAS, 323, 177

Topping, J. 1972, Errors of Observation and Their Treatment (Chapman and Hall), 62 